\title{
Search for dark forces with KLOE
}

\author{
Aleksander Gajos ${ }^{1, \star}$ \\ for the KLOE and KLOE-2 Collaborations \\ ${ }^{1}$ Institute of Physics, Jagiellonian University, \\ ul. Łojasiewicza 11, 30-348 Cracow, Poland
}

\begin{abstract}
During the last years several Dark Sector Models have been proposed in order to address striking astrophysical observations which failed standard interpretations. In the minimal case a new vector particle, the so called dark photon, or $A^{\prime}$ or $U$ boson, is introduced, with small coupling with Standard Model particles. Also, the existence of a dark Higgs boson $h^{\prime}$ is postulated, in analogy with the Standard Model, to give mass to the $U$ boson through the Spontaneous Symmetry Breaking mechanism. The KLOE experiment working at DAФNE, an $e^{+} e^{-}$collider operating in Frascati (near Rome), searched for the existence of the $U$ boson investigating three different processes and six different final states: (1) in Dalitz decays of the $\phi$ meson $\phi \rightarrow \eta U$, with $U \rightarrow e^{+} e^{-}$and $\eta \rightarrow \pi^{+} \pi^{-} \pi^{0}$ and $\pi^{0} \pi^{0} \pi^{0}$, (2) in $e^{+} e^{-} \rightarrow U \gamma$ events, with $U$ decaying into lepton and pion pairs, (3) in the dark Higgsstrahlung process, $e^{+} e^{-} \rightarrow U h^{\prime}, U \rightarrow \mu^{+} \mu^{-}, h^{\prime}$ invisible. Limits on the model parameters have been set at $90 \% \mathrm{CL}$ for $U$ boson masses below $1 \mathrm{GeV}$. Further improvements are expected in terms of sensitivity and discovery potential with the new KLOE-2 detector.
\end{abstract}

\section{Introduction}

Presently, dark matter investigations are one of the most active fronts of searches for physics beyond the Standard Model (SM). The introduction of Weakly Interacting Massive Particles as dark matter candidates would imply the presence of new interaction called the dark force and, consequently, a new gauge vector boson - the $U$ boson, also referred to as the dark photon or $A^{\prime}$. It would couple to the SM particles through a kinetic mixing parameter $\varepsilon$ equal to the ratio of dark and electromagnetic coupling constants. As $\varepsilon$ is expected to be in the range $10^{-4}-10^{-2}$, the dark photon should produce observable effects at $O(1 \mathrm{GeV})$ collider experiments such as KLOE at DAФNE. Moreover, search for the $U$ boson in such energy range is of special interest as it could explain a number of recent puzzing astrophysical observations such as the excess of positrons in cosmic rays observed by AMS and PAMELA [1, 2], $511 \mathrm{keV}$ gamma rays from the galactic centre seen by INTEGRAL [3] or the annual modulation signal measured by DAMA/LIBRA [4]. The KLOE experiment has searched evidence of the $U$ boson existence as a sharp resonance in the invariant mass spectrum of lepton or pion pairs produced in radiative processes $e^{+} e^{-} \rightarrow \ell^{+} \ell^{-}(\gamma)$ [5, 6], $e^{+} e^{-} \rightarrow \pi^{+} \pi^{-}(\gamma)$ [7] and in decays of the $\phi$ meson [8,9], as well as in the dark Higgsstrahlunng process [10].

^e-mail: aleksander.gajos@doctoral.uj.edu.pl 


\section{The KLOE detector at the DA $\Phi N E$ collider}

DAФNE is an $e^{+} e^{-}$collider located at the accelerator complex of LNF-INFN in Frascati, Italy. The center-of-mass energy of the colliding beams is typically set at the mass of the $\phi$ meson resonance $(\sqrt{s} \approx 1019 \mathrm{MeV})$ although measurements with off-peak energy are performed as well. The KLOE detector is shaped as a barrel around the $e^{+} e^{-}$collision point, constituted by a cylindrical drift chamber surrounded by an electromagnetic calorimeter and immersed in $0.52 \mathrm{~T}$ magnetic field. The drift chamber with a gas mixture of helium $(90 \%)$ and isobutane $(10 \%)$ provides position resolution of $150 \mu \mathrm{m}$ in the transverse plane and $2 \mathrm{~mm}$ in the $z$ direction whereas momentum of charged particles is reconstructed with a relative resolution of $0.4 \%$ [11]. The electromagnetic calorimeter is a sampling device based on scintillating fibers and lead which provides high efficiency for detection of $20-500 \mathrm{MeV}$ photons with an energy resolution of $5.7 \% E / \sqrt{E[\mathrm{GeV}]}$ and time resolution of $57 \mathrm{ps} / \sqrt{E[\mathrm{GeV}]} \oplus 100 \mathrm{ps}$ [12]. The detector has been recently upgraded and the new KLOE-2 experiment is collecting data with the aim to reach an integrated luminosity of about $5 \mathrm{fb}^{-1}$.

\section{Search for the $U$ boson in Dalitz decays of $\phi$}

A search for the $\mathrm{U}$ boson in the $\phi \rightarrow \eta U$ process, with $U$ decaying into an $e^{+} e^{-}$pair, has been performed at KLOE by analysing a data sample of $1.5 \mathrm{fb}^{-1}$ in the case of $\eta$ decaying into $\pi^{+} \pi^{-} \pi^{0}$ [9] and $1.7 \mathrm{fb}^{-1}$ in the case of $\eta \rightarrow \pi^{0} \pi^{0} \pi^{0}$ [8]. No evidence in the $e^{+} e^{-}$invariant mass spectrum was found for both searches [8,9]. A combined and more stringent upper limit [8] at $90 \%$ CL on the ratio between the coupling constant and the fine structure constant of $\alpha^{\prime} / \alpha<1.7 \times 10^{-5}$ for $30<m_{U}<$ $400 \mathrm{MeV}$ and $\alpha^{\prime} / \alpha<8 \times 10^{-6}$ for the sub-region $50<m_{U}<210 \mathrm{MeV}$ was evaluated. The result assumes the Vector Meson Dominance expectations for the $\phi \eta \gamma *$ transition form factor.

\section{Search for $e^{+} e^{-} \rightarrow U \gamma$ with $U$ decaying into lepton or pion pairs}

Evidence for the $e^{+} e^{-} \rightarrow U \gamma$ with $U \rightarrow \mu^{+} \mu^{-}$was searched in a sample of $239 \mathrm{pb}^{-1}$ in the $e^{+} e^{-} \rightarrow \mu^{+} \mu^{-} \gamma$ process with photon emitted in the initial state (ISR). The presence of the dark photon would be manifested as a peak in the recorded $m_{\mu \mu}$ mass distribution. Events were identified by two charged tracks at a large polar angle $\left(50^{\circ}<\theta<130^{\circ}\right)$ and an undetected photon at a small angle $\left(\theta<15^{\circ}\right.$ or $\left.\theta>165^{\circ}\right)$ which resulted in a strong suppression of the resonant $\phi \rightarrow \pi^{+} \pi^{-} \pi^{0}$ and FSR background. The residual background components were simulated and fitted to the di-muon mass spectrum. The experimental and simulated with PHOKHARA [13] $m_{\mu \mu}$ distributions have shown perfect agreement [6]. As no peak was observed in the spectrum, the $\mathrm{CL}_{S}$ technique was used to set a limit on the number of $U$ boson candidates and thus exclude $\varepsilon^{2}$ larger than $8.6 \times 10^{-7}-1.6 \times 10^{-5}$ for $520 \mathrm{MeV}<m_{U}<980 \mathrm{MeV}$ [6]. The obtained exclusion is marked as $\mathrm{KLOE}_{(2)}$ in Fig. 1.

A similar process, $e^{+} e^{-} \rightarrow U \gamma, U \rightarrow e^{+} e^{-}$was studied at KLOE in order to explore the region of low $U$ masses close to the di-electron mass threshold. In the analysis an explicit detection of both the electron and positron tracks and the photon was required. Dedicated analysis in order to distinguish electron-positron and muon couples was performed. Since the $e^{+} e^{-} \rightarrow e^{+} e^{-} \gamma$ process is dominant in the analysis acceptance, the total background was kept below $1 \%$. No peak in the $e^{+} e^{-}$invariant mass spectrum was observed and an upper limit on the kinetic mixing parameter was thus extracted. The obtained upper limit covers $m_{U}$ between $2 \mathrm{MeV}$ and $250 \mathrm{MeV}$ and ranges from $10^{-7}$ to $10^{-5}$ [5] (see the $\mathrm{KLOE}_{(3)}$ region in Fig. 1).

The last of the radiative processes investigated by KLOE was $e^{+} e^{-} \rightarrow U \gamma$ with $U \rightarrow \pi^{+} \pi^{-}$. The search was motivated by the lack of sensitivity of leptonic channels in the $\rho-\omega$ resonance mass 
region. The analysis was based on the one used for the measurements of the pion form factor [14] and a dedicated simulation was used based on the PHOKHARA generator [13] with the Gounaris-Sakurai pion form factor parametrization [15] to properly describe the $\rho-\omega$ region. No $U$ boson signal was observed and an upper limit on the $\varepsilon^{2}$ parameter was set for $m_{U}$ between 527 and $987 \mathrm{MeV}$. The excluded region indicated in Fig. 1 as $\mathrm{KLOE}_{(4)}$ shows a more stringent limit than the existing ones in the $\rho-\omega$ region and above [7].

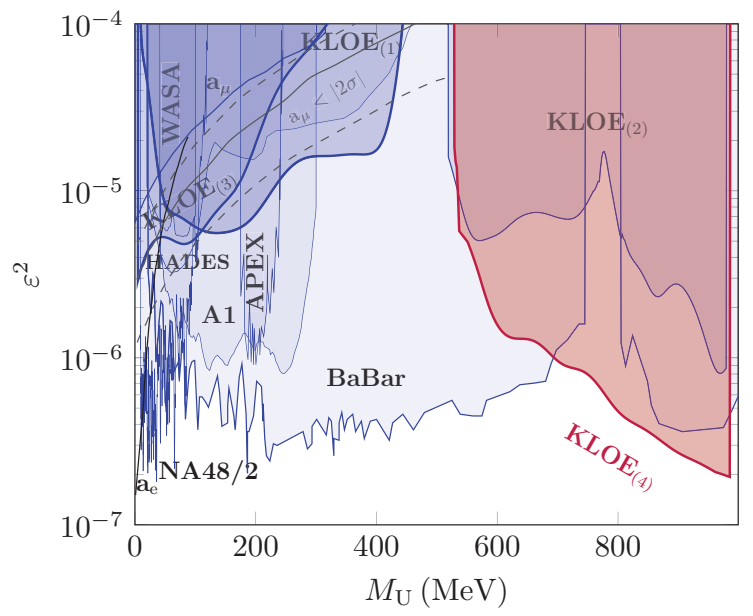

Figure 1. Exclusion limits (90\% CL) on the $\varepsilon^{2}$ parameter as a function of the $U$ boson mass. Presented are KLOE limits obtained with $\phi \rightarrow \eta e^{+} e^{-}\left(\mathrm{KLOE}_{(1)}\right), e^{+} e^{-} \rightarrow \mu^{+} \mu^{-} \gamma$ $\left(\mathrm{KLOE}_{(2)}\right), e^{+} e^{-} \rightarrow e^{+} e^{-} \gamma\left(\mathrm{KLOE}_{(3)}\right)$, $e^{+} e^{-} \rightarrow \pi^{+} \pi^{-} \gamma\left(\mathrm{KLOE}_{(4)}\right)$. For comparison the results of MAMI/A1 [16], APEX [17], WASA [18], HADES [19], NA48/2 [20] and BaBar [21] are given.

\section{Search for dark Higgsstrahlung}

Existence of the $U$ boson, as a massive particle, would imply breaking of its associated $\mathrm{U}_{D}$ symmetry through a Higgs-like particle $h^{\prime}$ called the dark Higgs. KLOE has searched for the dark Higgsstrahlung process $e^{+} e^{-} \rightarrow U h^{\prime}, U \rightarrow \mu^{+} \mu^{-}$in the "invisible dark Higgs" scenario where $h^{\prime}$ is lighter than $U$ and escapes detection due to its long lifetime. The analysed data included a $1.65 \mathrm{fb}^{-1}$ sample at the $\phi$ resonance peak and a $206 \mathrm{pb}^{-1}$ sample collected off-peak (at $1000 \mathrm{MeV}$ ). After identification of two muon tracks and reconstruction of the missing mass the signal was searched in a 2-dimensional $M_{m i s s}$ vs. $M_{\mu \mu}$ distribution. No resonance was observed and a bayesian upper limit on the number of candidate events was determined and translated to the $\alpha_{D} \times \varepsilon^{2}$ product where $\alpha_{D}$ is the coupling constant of the dark sector [10]. Resulting exclusion values depending on the $U$ and $h^{\prime}$ masses are presented in Fig. 2.

\section{Conclusions}

The KLOE experiment has made a large contribution to the searches of the dark forces mediator, the $U$ boson by exploring three different processes and a total of six final states. As no signal was observed by KLOE, upper limits on the kinetic mixing parameter $\varepsilon^{2}$ were set at the level between $10^{-7}$ and $10^{-5}$ (at $90 \% \mathrm{CL}$ ) in the $U$ boson mass range of 5-980 MeV. In the search for the dark Higgs with the $m_{h^{\prime}}<m_{U}$ scenario, the $\alpha_{D} \times \varepsilon^{2}$ product was limited at a $90 \% \mathrm{CL}$ in the range of $2 m_{\mu}<m_{u}<1000 \mathrm{MeV}$ and $10<m_{h^{\prime}}<500 \mathrm{MeV}$.

The KLOE-2 experiment with new subdetectors is presently taking new data with the aim to collect a sample of about $5 \mathrm{fb}^{-1}$. As the results reported here are presently limited by statistical uncertainty, KLOE-2 is expected to twice improve their sensitivity. 

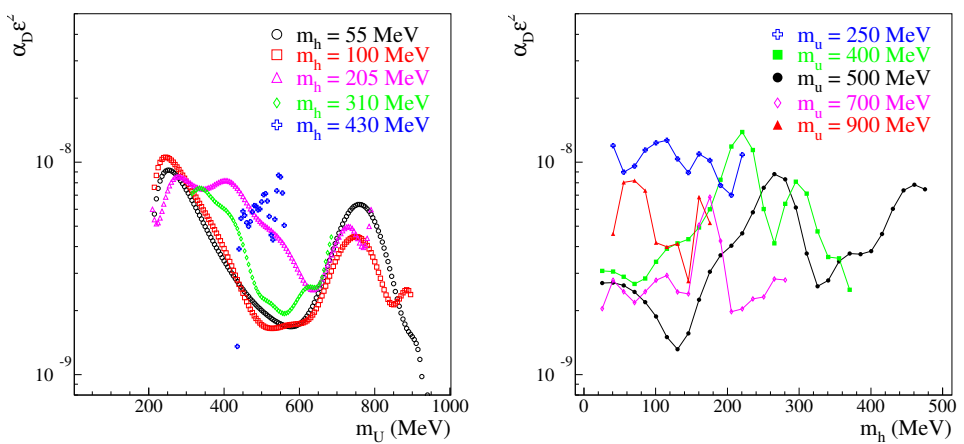

Figure 2. Upper limits $(90 \%$ CL) on $\alpha_{D} \times \varepsilon^{2}$ as a function of the $U$ boson mass for various values of the $h^{\prime}$ mass (left) and as a function of the $h^{\prime}$ mass for various values of $m_{U}$ (right) [10].

This work was supported in part by the EU Integrated Infrastructure Initiative Hadron Physics Project under contract number RII3-CT- 2004-506078; by the European Commission under the 7th Framework Programme through the 'Research Infrastructures' action of the 'Capacities' Programme, Call: FP7INFRASTRUCTURES-2008-1, Grant Agreement No. 227431; by the Polish National Science Centre through the Grants No. 2011/03/N/ST2/02652, 2013/08/M/ST2/00323, 2013/11/B/ST2/04245, 2014/14/E/ST2/00262, 2014/12/S/ST2/00459.

\section{References}

[1] M. Aguilar et al. (AMS), Phys. Rev. Lett. 110, 141102 (2013)

[2] O. Adriani et al. (PAMELA), Nature 458, 607 (2009)

[3] P. Jean et al., Astron. Astrophys. 407, L55 (2003)

[4] R. Bernabei et al. (DAMA, LIBRA), Eur. Phys. J. C 67, 39 (2010)

[5] A. Anastasi et al. (KLOE-2), Phys. Lett. B 750, 633 (2015)

[6] D. Babusci et al. (KLOE-2), Phys. Lett. B 736, 459 (2014)

[7] A. Anastasi et al. (KLOE-2), Phys. Lett. B 757, 356 (2016)

[8] D. Babusci et al. (KLOE-2), Phys. Lett. B 720, 111 (2013)

[9] F. Archilli et al. (KLOE-2), Phys. Lett. B 706, 251 (2012)

[10] A. Anastasi et al. (KLOE-2), Phys. Lett. B 747, 365 (2015)

[11] M. Adinolfi et al., Nucl. Instrum. Meth. A 488, 51 (2002)

[12] M. Adinolfi et al., Nucl. Instrum. Meth. A 482, 364 (2002)

[13] H. Czyz, A. Grzelinska, J.H. Kuhn, G. Rodrigo, Eur. Phys. J. C 39, 411 (2005)

[14] D. Babusci et al. (KLOE), Phys. Lett. B 720, 336 (2013)

[15] G.J. Gounaris, J.J. Sakurai, Phys. Rev. Lett. 21, 244 (1968)

[16] H. Merkel et al., Phys. Rev. Lett. 112, 221802 (2014)

[17] S. Abrahamyan et al. (APEX), Phys. Rev. Lett. 107, 191804 (2011)

[18] P. Adlarson et al. (WASA-at-COSY), Phys. Lett. B 726, 187 (2013)

[19] G. Agakishiev et al. (HADES), Phys. Lett. B 731, 265 (2014)

[20] J.R. Batley et al. (NA48/2), Phys. Lett. B 746, 178 (2015)

[21] J.P. Lees et al. (BaBar), Phys. Rev. Lett. 113, 201801 (2014) 